\title{
Study of impact of diabetes mellitus on corneal central thickness and endothelial parameters in Indian eyes
}

\author{
Shankar C ${ }^{1}$, Premnath $G^{2, *}$, Balaji A ${ }^{3}$, Lakshmi Ananya Aila ${ }^{4}$, Sruthi $G^{5}$ \\ ${ }^{1,2}$ Associate Professor, ${ }^{3}$ Professor \& HOD, ${ }^{4,5}$ Post Graduate, Dept. of Ophthalmology, ${ }^{\mathbf{1 , 2 , 4 , 5}}$ Chettinad Academy of Research and \\ Education, Kelambakkam, Tamil Nadu, India, ${ }^{3}$ Tagore Medical College \& Hospital, Tamil Nadu, India
}

*Corresponding Author:

Email: drprem1822@gmail.com

\begin{abstract}
Aim: The purpose of the study is to find the impact of diabetes mellitus on central thickness and endothelial parameters of cornea when compared with non diabetic patients and in relation to duration of disease and glycaemic control.

Study Design: Prospective, Observational and quantitative study.

Materials and Methods: Study included 125 patients (125 eyes) with diabetes mellitus and 143 controls (143 eyes). Tomey specular non-contact microscope (EM 3000) was used to measure central corneal thickness (CCT), endothelial cell density (ECD), coefficient of variation of cell size (CV) and percentage of hexagonal endothelial cells (6A).

Results: Comparison of age matched controls with diabetics did not show any statistical significance with respect to CCT, ECD and CV. 6A showed some significant change $(\mathrm{p}$ value $=0.02)$ between controls and diabetics. There was no significant correlation with respect to the duration of diabetes and glycaemic control.

Conclusion: Corneal endothelial parameters and thickness did not show any statistical significance between diabetics and controls except hexagonality of endothelial cells which was significant. Moreover there was statistically no difference based on duration of the disease, glycaemic control and presence or absence of retinopathy.
\end{abstract}

Keywords: Central corneal thickness, Endothelial cell density, Diabetes mellitus.

\section{Introduction}

Diabetes mellitus is a major systemic disease in our society and its prevalence is increasing day by day significantly. At the ocular level, main indicators for diabetes are diabetic retinopathy, cataract and glaucoma. Diabetic retinopathy is the 5th leading cause of blindness globally, while it is the first leading cause of blindness in the developed countries in adults at working age. Though the cornea appears normal, significant biochemical changes occur which alter the function of cornea. Advanced glycation end products $(\mathrm{AGEs})^{1-4}$ and matrix metalloproteinases (MMP) have been proposed to be the cause for corneal damage. The accumulation of AGEs enhanced the apoptosis of retinal pericytes, corneal endothelial cells, neuronal cells, and renal mesangial cells. AGEs is seen in the corneal stroma, Descemet's membrane and basement membrane of the corneal epithelium. The accumulation of the AGEs in diabetic corneas provided evidence that nuclear oxidative DNA damage caused by AGEs is responsible for the apoptotic damage of cornea in diabetic patients ${ }^{5}$ Na-K ATPase of cornea endothelium is also affected which causes change in permeability of cornea. ${ }^{6}$

Corneal thickness increases in diabetic patients possibly due to inhibition of endothelial pump, increased endothelial permeability and increased swelling pressure of corneal stroma. ${ }^{6}$ The purpose of the study is to find the impact of diabetes mellitus on central corneal thickness (CCT), endothelial count (ECD), CV and hexagonality in relation to duration of disease and glycaemic control.

\section{Materials and Methods}

This is a prospective, observational quantitative study done in Chettinad Hospital and Research institute. It adhered to the tenants of Declaration of Helsinki. Comprehensive consent was obtained after explaining the study. The study included 125 diabetic patients (Male- 66, Female- 59) and 143 controls (Male- 61, Female- 82).

Inclusion Criteria: All diabetic patients aged 30 years and above consented for the study were included.

Exclusion Criteria: All patients with any history of ocular surgery, trauma, corneal diseases, congenital anomalies of eye, previous eye infection or inflammation, glaucoma, ocular laser procedures, pterygium, lid anomalies, regular usage of contact lens, eye drops, systemic drugs interfering with tear film were excluded. Patients with systemic illness interfering with tear film like rheumatoid arthritis, systemic lupus erythematosis were also excluded.

A complete ophthalmic examination and relevant laboratory investigations were done. Tomey non-contact specular microscope (EM 3000) was used to evaluate corneal parameters. Average of three readings were taken after instilling lubricant eye drops. $\mathrm{CCT}$, ECD, polymegathism (CV) and \% of hexagonal cells (6A) were taken for study. All the information were recorded in a precoded format. 


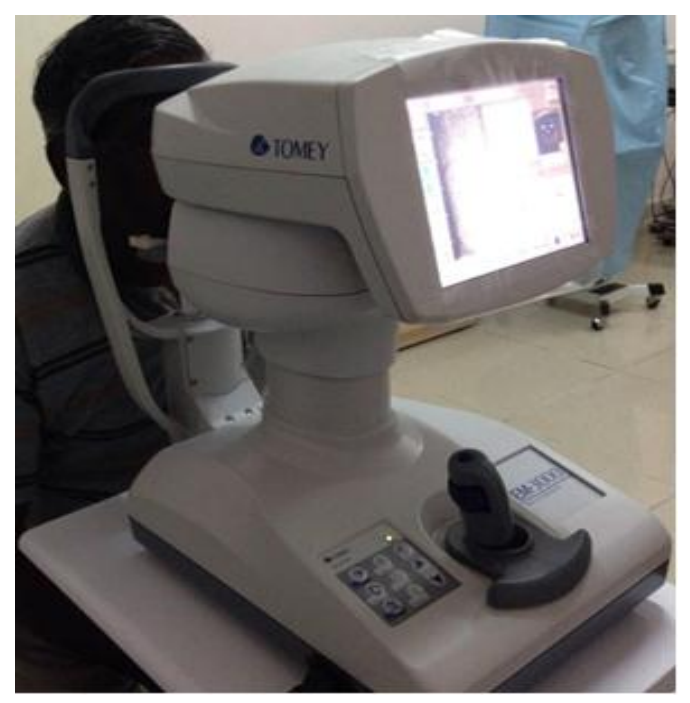

Fig. 1: Measurement of corneal central thickness and endothelial parameters

The diabetics were divided into two groups each based on

1. Duration of disease i.e Less than 5 years and greater than 5 years
2. Glycemic control i.e HbA1c less than $7 \mathrm{gm} / \mathrm{dl}$ and greater than $7 \mathrm{gm} / \mathrm{dl}$,

3. Age of the diabetics i.e less than 50 years old and above 50 years

4. With and without retinopathy.

Statistical Analysis: Unpaired t test was used for statistical analysis to compare controls with diabetics first and then comparison among diabetic subgroups were done.

\section{Results}

Mean age of diabetic patients was $53.84 \pm 10.80$ years and controls $53 \pm 9.29$ years.

Table 1 shows the comparison of age matched controls with diabetics which did not show any statistical significance with respect to CCT, ECD and CV. Hexagonality of endothelial cell showed statistically significant change $(p$ value $=0.02)$ between controls and diabetics.

Table 1: Control Vs diabetic patients

\begin{tabular}{|c|c|c|c|}
\hline Corneal parameters & Controls $(n=143)$ & Diabetics $(n=125)$ & P value \\
\hline CCT & $521.57+48.78$ & $518.1 \pm 43.15$ & 0.584 \\
\hline ECD & $2504.41+305.25$ & $2529.4 \pm 365.30$ & 0.542 \\
\hline $\mathrm{CV}$ & $40.14+10.74$ & $40.48 \pm 10.89$ & 0.820 \\
\hline $6 \mathrm{~A}$ & $45.4 \pm 7.81$ & $43.5 \pm 5.92$ & 0.020 \\
\hline
\end{tabular}

Comparison of controls with duration of diabetes greater than 5 years did not show any statistical significance (Table-2).

Table 2: Control Vs Duration of diabetes $>5$ yrs

\begin{tabular}{|l|c|c|c|}
\hline Corneal parameters & Controls $(\mathbf{n = 1 4 3})$ & Diabetes more than 5yrs $(\mathbf{n = 6 0})$ & P value \\
\hline CCT & $521.57+48.78$ & $513.7 \pm 49.2$ & 0.301 \\
\hline ECD & $2504.41+305.25$ & $2574.6 \pm 253.3$ & 0.119 \\
\hline CV & $40.14+10.74$ & $40.1 \pm 8.7$ & 1.00 \\
\hline 6A & $45.4 \pm 7.81$ & $43.8 \pm 6.2$ & 0.303 \\
\hline CCT-Central corneal thickness, ECD-Endothelial cell density, CV-Co-efficient of variation, 6A- \\
Percentage of hexagonal cells
\end{tabular}

Comparison of the parameters with controls and diabetics with HbA1c $>7$ did not show any significance (Table 3 ). Comparison with controls and diabetic with retinopathy also showed no significance (Table 4).

Table 3: Control Vs HbA1c $>7$

\begin{tabular}{|l|c|c|c|}
\hline Corneal parameters & Controls $(\mathbf{n}=143)$ & HbA1c $>$ 7 $(\mathbf{n}=\mathbf{1 0 8})$ & P value \\
\hline CCT & $521.57+48.78$ & $518.1 \pm 44.6$ & 0.571 \\
\hline ECD & $2504.41+305.25$ & $2524.2 \pm 382.5$ & 0.645 \\
\hline CV & $40.14+10.74$ & $39.5 \pm 8.84$ & 0.694 \\
\hline 6A & $45.4 \pm 7.81$ & $43.4 \pm 6.1$ & 0.079 \\
\hline
\end{tabular}

CCT-Central corneal thickness, ECD-Endothelial cell density, CV-Co-efficient of variation, 6A-

Percentage of hexagonal cells 
Table 4: Control Vs diabetic retinopathy

\begin{tabular}{|l|c|c|c|}
\hline Corneal parameters & Controls $(\mathbf{n = 1 4 3})$ & $\begin{array}{c}\text { Diabetic patients with } \\
\text { retinopathy }(\mathbf{n = 2 9})\end{array}$ & P value \\
\hline CCT & $521.57+48.78$ & $515.3 \pm 38.3$ & 0.500 \\
\hline ECD & $2504.41+305.25$ & $2539.2 \pm 219.3$ & 0.558 \\
\hline CV & $40.14+10.74$ & $40.3 \pm 7.0$ & 1.000 \\
\hline 6A & $45.4 \pm 7.81$ & $42.6 \pm 5.9$ & 0.119 \\
\hline $\begin{array}{l}\text { CCT-Central corneal thickness, ECD-Endothelial cell density, CV-Co-efficient of variation, 6A- } \\
\text { Percentage of hexagonal cells }\end{array}$
\end{tabular}

After comparison of diabetics with controls, the corneal parameters were compared within the diabetic group (Table 5). Among diabetic patients, $\mathrm{CV}$ alone showed significance when they were compared in terms of age less than 50 years and more than 50 years and the glycemic control. Other parameters did not show any significance.

Table 5: Comparison of corneal parameters within diabetic group

\begin{tabular}{|c|c|c|c|c|}
\hline Diabetic sub groups & CCT & ECD & CV & 6A \\
\hline Age $<50$ yrs $(n=52)$ & $522.75 \pm 35.45$ & $2517.71 \pm 384.37$ & $41.89 \pm 13.72$ & $44.00 \pm 6.44$ \\
\hline Age $>50 y r s(n=73)$ & $514.8 \pm 47.86$ & $2537.75 \pm 353.56$ & $39.48 \pm 8.3$ & $43.29 \pm 5.55$ \\
\hline $\mathrm{P}$ value & $0 . \overline{383}$ & 0.779 & 0.012 & 0.323 \\
\hline Duration $<5$ yrs $(\mathrm{n}=65)$ & $522.14 \pm 36.56$ & $2487.66 \pm 442.39$ & $40.82 \pm 12.64$ & $43.35 \pm 5.63$ \\
\hline Duration > 5yrs $(\mathrm{n}=60)$ & $513.73 \pm 49.26$ & $2574.65 \pm 253.31$ & $40.12 \pm 8.71$ & $43.83 \pm 6.26$ \\
\hline $\mathrm{P}$ value & $0 . \overline{525}$ & $0 . \overline{436}$ & 0.380 & $0 . \overline{651}$ \\
\hline $\operatorname{HbA1C}<7 \mathrm{gm} \%(\mathrm{n}=17)$ & $517.655+33.64$ & $2562.24 \pm 233.9$ & $46.65 \pm 18.71$ & $44.58+4.58$ \\
\hline $\mathrm{HbA1C}>7 \mathrm{gm} \%(\mathrm{n}=108)$ & $518.18 \pm 44.6$ & $2524.25 \pm 382.45$ & $39.50 \pm 8.85$ & $43.42 \pm 6.11$ \\
\hline $\mathrm{P}$ value & 0.431 & 0.601 & 0.001 & 0.266 \\
\hline Retinopathy-No (n=29) & $515.34+38.30$ & $2539.28 \pm 219.30$ & $40.31 \pm 7.08$ & $42.55+5.94$ \\
\hline Retinopathy-Yes $(n=96)$ & $518.94 \pm 44.67$ & $2526.44 \pm 399.96$ & $40.53 \pm 11.84$ & $43.9 \pm 5.91$ \\
\hline P value & 0.917 & 0.362 & $0 . \overline{6} 45$ & $0 . \overline{801}$ \\
\hline
\end{tabular}

\section{Discussion}

Corneal endothelial parameters change in diabetics is well observed in literature and articles, showing reduced endothelial density, increased polymegathism (CV), decrease in hexagonality of cells and increased corneal thickness. ${ }^{7,8}$ According to our study this was not the observation, ECD, CV and corneal thickness did not show any significant change between diabetics and controls. Hexagonality of cells alone had significant difference $(p=0.02)$. This is similar to a study done by Sudhir et $\mathrm{al}^{9}$ and Shashi et $\mathrm{al}^{10}$ who showed that there was no difference observed in CCT, CV and hexagonality between diabetics and controls, but they showed reduced ECD in diabetics. In our study ECD was not statistically different from control, this could be due to increased deviation of cell count between diabetics and controls. (SD in diabetics is 365 compared to 305 in controls).

Corneal endothelial parameters were also compared with age of patient, duration of diabetes, glycaemic control and presence of diabetic retinopathy. JS Lee et $\mathrm{al}^{7}$ has shown that patients with diabetes of more than 10 years showed significant difference in ECD, CV, CCT and hexagonality. In our study there was no significant difference in CCT, CV, ECD and hexagonality. This was similar to a study done by Stella et $\mathrm{al}^{11}$ who showed there was no correlation with disease duration. In our study comparison was between less than 5 years and above 5 years, but most of the studies compared 10 years of diabetes.

Comparison of glycaemic control in relation with HbAlc below and above 7 showed no significant correlation with respect to CCT, ECD and hexagonality but CV showed significance. This is similar to Vineetha et $\mathrm{al}^{12}$ who showed HbA1c had negative correlation with ECD and hexagonality of cells. However studies by Allanstor et $\mathrm{al}^{13}$ and Manish Gupta et $\mathrm{al}^{14}$ showed increase in $\mathrm{HbAlc}$ greater than $7 \mathrm{gm} / \mathrm{dl}$ was associated with changes in CCT and endothelial parameters. The variation in our study could be due to the number of patients compared. (Patients with HbAlc less than 7 was 17 and above 7 was 108). Neither age nor the presence or absence of retinopathy showed any significant correlation.

\section{Conclusion}

In conclusion corneal endothelial parameters and thickness did not show any statistical significant difference between diabetics and controls except hexagonality of endothelial cells which was significant. Moreover there was statistically no difference based on duration of the disease, glycaemic control and presence 
or absence of retinopathy.

\section{References}

1. Kaji Y et al. Advanced glycation end products in diabetic corneas. Invest Ophthalmol Vis

Sci. 2000;41:362-368.[PubMed]

2. Sato E, Mori F, Igarashi S, Abiko T, Takeda M, Ishiko S, Yoshida A. Corneal advanced glycation end products increase in patients with proliferative diabetic retinopathy. Diabetes Care. 2001;24:479-482. [PubMed]

3. Choo M, Prakash K, Samsudin A, Soong T, Ramli N,

Kadir A. Corneal changes in type II diabetes mellitus in Malaysia. Int J Ophthalmol. 2010;3(3):234-236.

4. Alexander ljubiniov. Diabetic complications in the cornea. Vision research. Volume 139. October 2017:138-152.

5. Guzel Bikbova et al, Corneal Changes in Diabetes Mellitus article review in Current diabetes reviews 8 (4) 294-302, May 2012.

6. Joseph A Bonanno, Molecular mechanism underlying the corneal endothelial pump, Exp Eye research 2012, Feb 95(1) 2-7.

7. Lee JS, Oum BS, Choi HY, et al. Differences in corneal thickness and corneal endothelium related to duration in diabetes[J] Eye. 2006;20:315-318. [PubMed]

8. El-Agamy A. Corneal Endothelium and Central Corneal Thickness Changes in type 2 Diabetes Mellitus. Ins Ophthal. 2017, 1:2.

9. Sudhir R, Raman R, Sharma T. Changes in the corneal endothelial cell density and morphology in patients with type 2 diabetes mellitus: a population-based study, Sankara Nethralaya Diabetic Retinopathy and Molecular Genetics Study (SN-DREAMS, Report 23) Cornea. 2012;31(10):1119-1122.

10. Shashi et al, Corneal endothelial changes in patients with Type 2 diabetes using specular microscopy. EC ophthalmology 6.4(2017), 100-107.

11. Stella Briggs et al, Manifestation of type 2 diabetes in corneal endothelial density, corneal thickness and IOP, The journal of bio medical research. 2016. Jan 30 (1):46-51.

12. Vineetha Prakasam et al. Alteration in corneal structures in patients with diabetes. Journal of medical science and research. 2017. August. Vol 5, Issue 8: 27140-27144.

13. Allan Storr Paulsen et al. Corneal endothelial morphology and central corneal thickness in patients with type II diabetes, Acta Ophthalmologia 2014, 92, 158-160.

14. Manish Gupta et al, A study of corneal changesendothelial cell density and corneal thickness in type II diabetes in relation to HbA1c and compare with healthy individuals. Indian journal of clinical and experimental ophthalmology. April- June 2016, 123-127. 